\title{
The pocket-creation method facilitates endoscopic submucosal dissection of gastric neoplasms involving the pyloric ring $\square$
}

\section{(ㄷ)(i) $\Theta$}

\author{
Authors \\ Masafumi Kitamura', Yoshimasa Miura' ${ }^{1}$, Satoshi Shinozaki ${ }^{1,2}$, Hirotsugu Sakamoto ${ }^{1}$, Yoshikazu Hayashi ${ }^{1}$, Mio \\ Sakaguchi $^{3}$, Noriyoshi Fukushima ${ }^{3}$, Alan Kawarai Lefor ${ }^{4}$, Hironori Yamamoto ${ }^{1}$
}

Institutions

1 Department of Medicine, Division of Gastroenterology, Jichi Medical University, Shimotsuke, Japan

2 Shinozaki Medical Clinic, Utsunomiya, Japan

3 Department of Diagnostic Pathology, Jichi Medical University, Shimotsuke, Japan

4 Department of Surgery, Jichi Medical University, Shimotsuke, Japan

submitted 8.10 .2020

accepted after revision 3.2.2021

Bibliography

Endosc Int Open 2021; 09: E1062-E1069

DOI 10.1055/a-1403-1153

ISSN 2364-3722

(C) 2021. The Author(s).

This is an open access article published by Thieme under the terms of the Creative Commons Attribution-NonDerivative-NonCommercial License, permitting copying and reproduction so long as the original work is given appropriate credit. Contents may not be used for commercial purposes, or adapted, remixed, transformed or built upon. (https://creativecommons.org/licenses/by-nc-nd/4.0/)

Georg Thieme Verlag KG, Rüdigerstraße 14,

70469 Stuttgart, Germany

Corresponding author

Hironori Yamamoto, MD, PhD, 3311-1 Yakushiji, Shimotsuke, Tochigi 329-0498, Japan

Fax: +81-285- 40-6598

ireef@jichi.ac.jp

\section{ABSTRACT}

Background and study aims Endoscopic submucosal dissection (ESD) of superficial gastric lesions involving the pyloric ring is difficult. The pocket-creation method (PCM) with a small-caliber-tip transparent hood can overcome this difficulty by compressing the pyloric sphincter applying both traction and counter-traction. The aim of this study is to clarify the usefulness of the PCM for ESD of superficial gastric neoplasms involving the pyloric ring compared to the conventional method (CM).

Patients and methods From October 2006 to August 2019, 66 gastric lesions requiring duodenal submucosal dissection beyond the pyloric ring in 66 patients were resected. The $\mathrm{CM}$ was mainly performed in the first period (CM group, $n=46$ ) and the PCM in the second period (PCM group, $n=20$ ). We retrospectively reviewed their medical records.

Results Although no significant differences were observed in en bloc resection rates between the two groups, the PCM group had a significantly higher $\mathrm{R} 0$ resection rate than the CM group ( $P=0.047)$. There were no holes in resected specimens in the PCM group while three specimens in the CM group had a hole. The dissection speed in the PCM group tended to be higher than in the CM group, although it did not reach statistical significance $(P=0.148)$. No significant differences were observed for the incidence of adverse events.

Conclusions This is the first study reporting the advantages of the PCM over the CM for ESD of gastric lesions involving the pyloric ring. We believe that the PCM is an effective strategy to compress the pyloric sphincter and facilitates R0 resection.

\section{Introduction}

Gastric cancer is a common neoplasm worldwide and was the second most common malignancy causing death in 2018 following lung cancer [1]. Since the evolution of endoscopy, including image enhanced endoscopy, facilitates the early detection of gastric cancer, indications for minimally invasive resec- tion are expanding. Endoscopic submucosal dissection (ESD) is one of the standard endoscopic treatments for superficial gastric neoplasms. However, the difficulty associated with performing ESD of gastric lesions varies greatly depending on the location of the lesion because the stomach has a wide lumen and unique shape. ESD of gastric lesions in difficult locations increases the risk of adverse events and prolongs procedure time 
due to slow rate of dissection. Gastric neoplasms involving the pyloric ring is one of the difficult situations encountered in ESD of gastric lesions [2]. The working space around the pyloric ring is limited. At the duodenal bulb, the presence of Brunner's glands and poor mucosal elevation after injection make ESD difficult.

In the conventional method (CM), an antegrade approach creates a vertical approach to the pyloric sphincter and the submucosal side of the dissected mucosa. After getting over the pyloric ring, a horizontal approach in the duodenal bulb is difficult due to the firm and high muscularis at the pyloric ring ( $>$ Fig.1a). Retroflexion in the duodenal bulb is one option to overcome this difficulty. However, a vertical approach to the duodenal wall is inevitable in the area near the pyloric sphincter. Poor maneuverability during retroflexion has the potential to cause perforation due to the thin wall and narrow lumen of the duodenum ( $>$ Fig. $\mathbf{1 b}$ ). Further, retroflexion requires a slim endoscope with a small working channel that restricts the kind of devices that can be introduced. A traction device such as the clip-and-line method has been reported to overcome these difficulties [3]. However, traction cannot facilitate opening the submucosal layer in the duodenum, because it cannot compress the pyloric sphincter but just pulls the lesion.

We invented the pocket-creation method (PCM) to overcome the difficulties associated with ESD and reported its usefulness in performing stable and reliable ESD $[4,5]$. In short, the PCM is characterized by a minimal mucosal incision and making a pocket without a circumferential incision. The PCM has four major advantages: (1) preventing leakage of injected solution, (2) applying both traction and counter-traction, (3) conquering the tangential approach and (4) stabilization of the tip of the endoscope in the pocket [4]. We believe that the PCM is especially helpful to overcome the above-mentioned difficult circumstances associated with ESD of superficial gastric lesions in-


performing ESD of gastric lesions involving the pyloric ring, compressing the pyloric sphincter is the key to accomplishing an en bloc resection. Compression can be achieved by providing traction and counter-traction using the PCM with a small-caliber-tip transparent hood (ST-hood). The PCM prevents migration of the mucosa being dissected into the duodenum due to a minimal mucosal incision and enables antegrade straightforward submucosal dissection even in the duodenum without retroflexion. The aim of this study was to clarify usefulness of the PCM for ESD of superficial gastric neoplasms requiring duodenal submucosal dissection beyond the pyloric ring compared to the CM.

\section{Patients and methods}

\section{Study population}

From October 2006 to August 2019, 2258 gastric lesions were treated with ESD at Jichi Medical University Hospital. Of these 2258 lesions, 66 lesions in 66 patients required duodenal submucosal dissection beyond the pyloric sphincter. We retrospectively reviewed the medical records of these patients. The PCM was introduced in September 2014. The CM was performed



- Fig. 1 The conventional method was performed as follows. a An antegrade approach gives a vertical approach at the pyloric sphincter. To avoid the solid muscularis of the pyloric sphincter, the upward angle also leads to a vertical approach against the submucosal side of the dissected mucosa that may damage the resected specimen. After getting over the pyloric ring, a horizontal approach in the duodenal bulb is impossible because the dissected mucosa falls down to the duodenal side and the tip of the endoscope becomes unstable due to the high muscularis of the pyloric ring. b With a retroflexed approach, maneuverability is limited in the narrow space in the duodenal bulb. The vertical approach at the pyloric sphincter makes submucosal dissection difficult.

during the first period (October 2006 to August 2014) except in two patients (CM group, $n=46$ ) and the PCM was performed during the second period (September 2014 to August 2019, PCM group, $n=20$ ) ( $\mathbf{F i g . 5}$ ). We compared the PCM group to the CM group (control group). Informed consent regarding ESD was obtained. The Institutional Review Board approved this retrospective review (ID\#A19-255). 


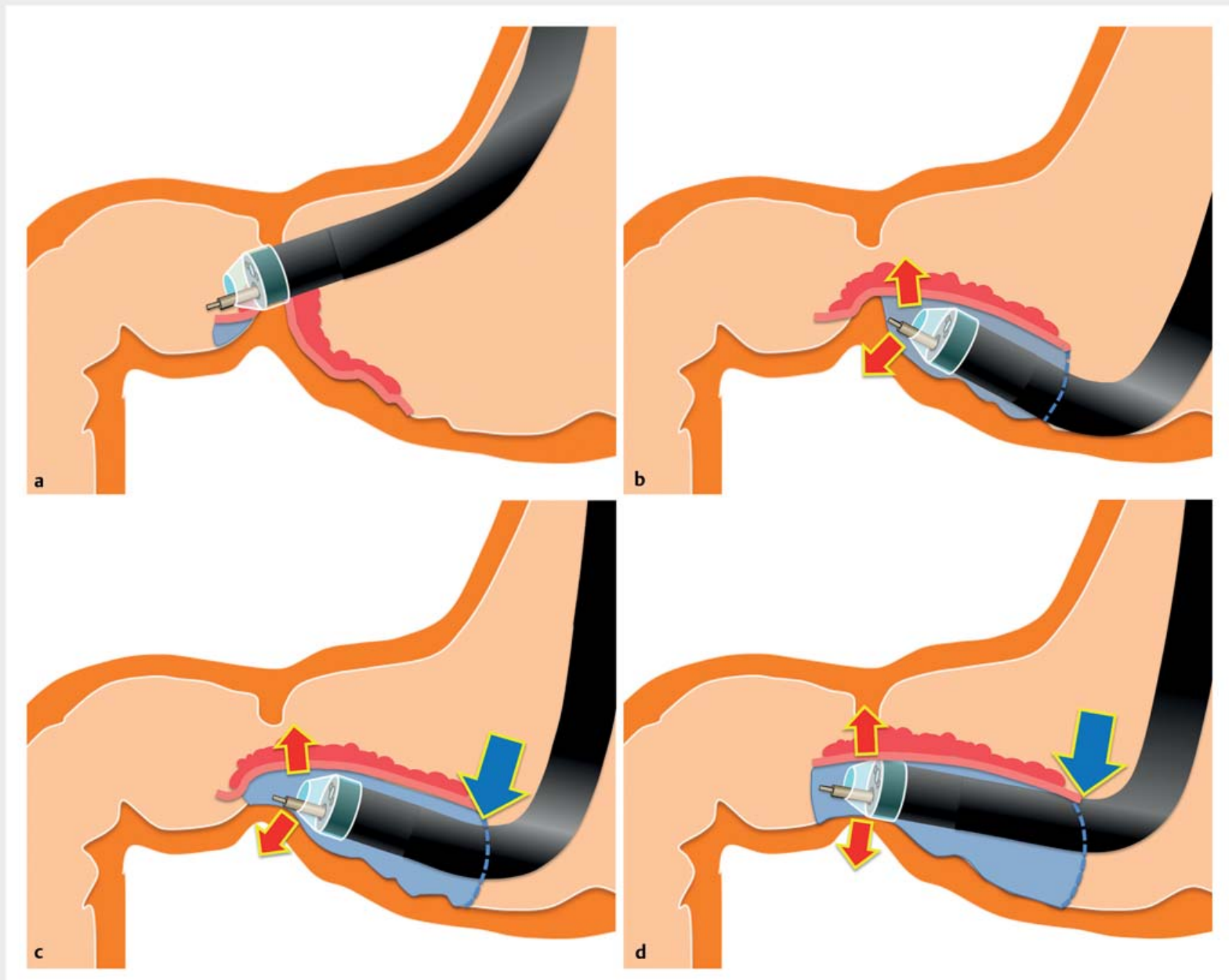

Fig. 2 The pocket-creation method with an ST hood is performed as follows. a The duodenal side of the tumor is incised without submucosal dissection. $\mathbf{b}$ The gastric side is minimally incised and then dissected to make a pocket. $\mathbf{c}$ At the pyloric ring, stable and well-visualized submucosal dissection is enabled by compressing the pyloric sphincter using both traction and counter-traction (red arrows). The tip of the endoscope is effectively held by the pocket (blue arrow). $\mathbf{d}$ A tunnel is created from the gastric to the duodenal sides in an antegrade manner.

\section{Endoscopic resection}

All patients were hospitalized and underwent ESD under conscious sedation with pethidine and midazolam. All ESD procedures were performed by expert endoscopists, defined as an endoscopist with an experience of having performed more than 50 ESD procedures of gastric lesions. An endoscope with a waterjet instrument (EG-450RD or EG-580RD, Fujifilm, Tokyo, Japan) was used for submucosal dissection without retroflexion. If retroflexion was required in the CM, an endoscope (GIFXQ240, Olympus, Tokyo, Japan) was used for marking, mucosal incision and submucosal dissection in the duodenum. In the $P C M$, retroflexion was not required in any cases. Carbon dioxide insufflation was used. A small-caliber-tip transparent (ST) hood (DH-15GR [conventional ST hood] or DH-28GR [short ST hood], Fujifilm) or a cylindrical hood (D-201-10804, Olympus) fitted to the tip of the endoscope was used. The injection solution was $0.4 \%$ sodium hyaluronate (MucoUp, Seikagaku, Tokyo, Japan) with $0.002 \%-0.004 \%$ indigo carmine and $0.001 \%$ epinephrine. We used a Flush knife BT (DK2618JB-15, Fujifilm), a DualKnife (KD-650Q, Olympus), a SAFE Knife V (DK2518DV15, Fujifilm) or a needle knife (KD-10Q-1, Olympus). To control bleeding during the ESD procedure, hot hemostatic forceps (HOYA Corporation, Tokyo, Japan) were used. The electrosurgical generator used was an ICC 200 or a VIO300 D (ERBE Elektromedizin GmbH, Tübingen, Germany).

En bloc resection was defined as endoscopic resection yielding a single piece macroscopically. An R0 resection was defined as an en bloc resection with negative pathological margins for both adenoma and adenocarcinoma. The area of the resected specimen was calculated with the following formula: major axis $(\mathrm{mm}) / 2 \times$ minor axis $(\mathrm{mm}) / 2 \times 3.14$. The dissection time was defined as the time between the initial mucosal incision and completion of the resection. The dissection speed was calculated with the following formula: specimen area $\left(\mathrm{mm}^{2}\right) /$ dis- 


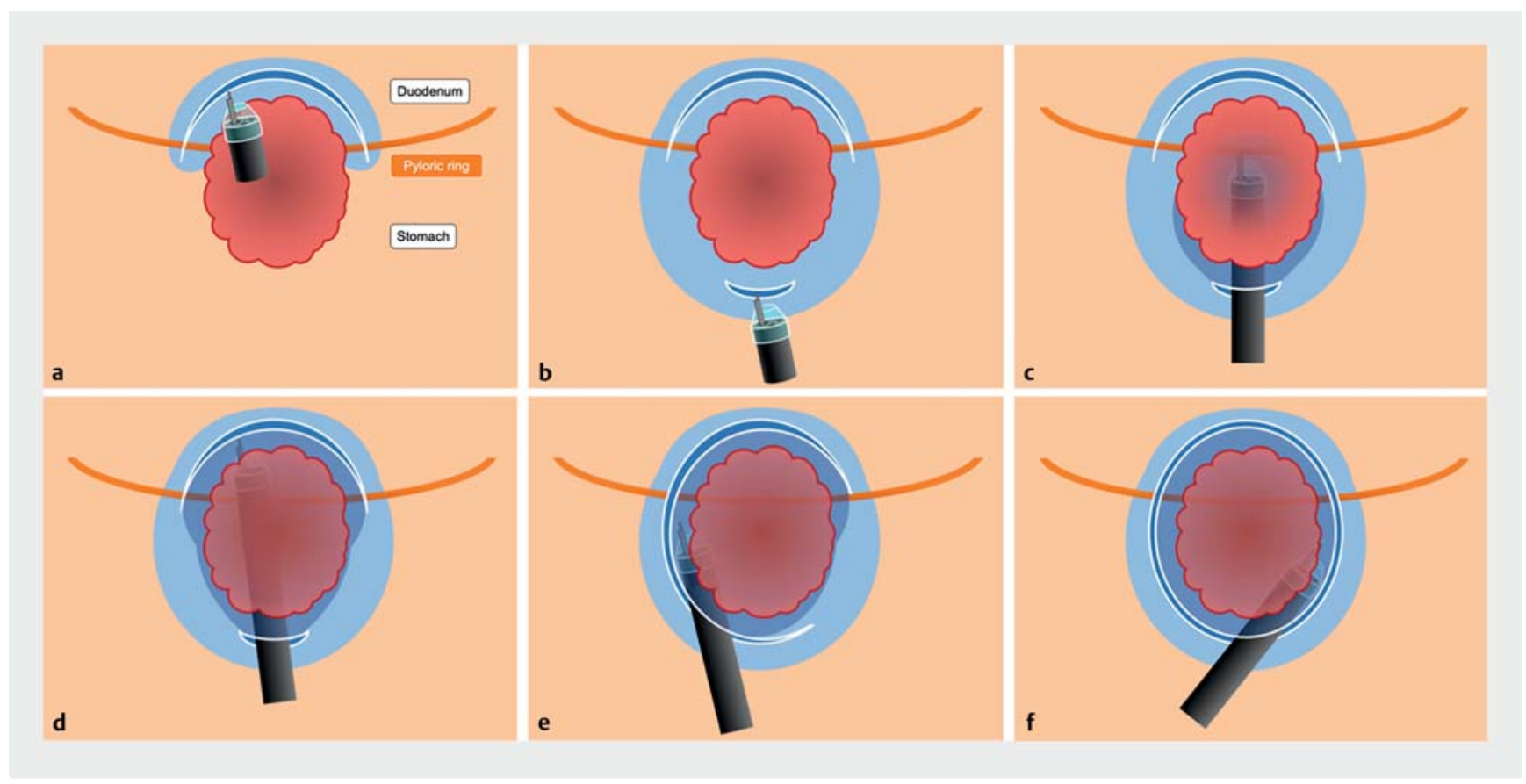

- Fig. 3 Schematic of the pocket-creation method viewed from above. a A mucosal incision is made on the duodenal side of the tumor to the pyloric ring. b A minimal mucosal incision is made on the gastric side. c A large submucosal pocket is created under the tumor while compressing the pyloric sphincter. $\mathbf{d}$ A tunnel is created from the gastric to the duodenal sides in an antegrade manner. Submucosal dissection under the lesion should be completed before opening the pocket. e The pocket is opened in a step-by-step manner working toward the distal side and against gravity. $\mathrm{f}$ The remaining area is dissected and an en bloc resection is accomplished.

section time (minutes) [6]. Intraprocedural and delayed perforations were defined as perforations during or after the ESD procedure, respectively. Delayed bleeding was defined as melena or hematochezia with a decrease in hemoglobin concentration of more than $2 \mathrm{~g} / \mathrm{dL}$, requiring transfusion or requiring endoscopic hemostasis within 14 days after ESD [7]. The primary outcome measure was the R0 resection rate, and secondary outcome measures included the en bloc resection rate, presence of holes in the resected specimens, positive horizontal/vertical margins, dissection speed, requirement for snare use and the incidence of adverse events.

\section{Statistical analysis}

Stata software version 16 (StataCorp LLC, Texas, United States) was used for analysis, and differences were considered significant when $P<0.05$. Data without a normal distribution were evaluated using the Mann-Whitney U-test. Categorical data were assessed with the chi-square test.

\section{Results}

\section{Baseline characteristics of patients}

There were no differences between the PCM and CM groups in baseline characteristics such as location, macroscopic type and size except for the presence of an ulcer ( $\bullet$ Table 1$)$. A conventional ST hood was used more often than in the PCM group compared to the CM group, because the ST hood is essential for the PCM to obtain both traction and counter-traction in the pocket. Traction devices were not used in any procedure.

\section{Quality of ESD}

Although no significant differences were observed in en bloc resection rates between the two groups, the PCM group had a significantly higher $\mathrm{R} 0$ resection rate than the $\mathrm{CM}$ group $(P=$ 0.047) ( $\triangleright$ Table 2 ). There were no holes in the resected specimens in the PCM group while three lesions (7\%) had a hole in the CM group. The dissection speed in the PCM group was faster than in the CM group, although this difference did not reach

- Fig.4 An example of endoscopic submucosal dissection with the pocket-creation method. a A gastric elevated lesion involving the pyloric ring observed from the gastric side. $\mathbf{b}$ Observed from the duodenal side with retroflexion. $\mathbf{c}$ First mucosal incision at the distal side in the duodenal bulb. $\mathbf{d}$ A minimal mucosal incision of the proximal side in the stomach. e Antegrade approach compressing the pyloric sphincter using both traction and counter-traction by the tip of the ST-hood. $\mathbf{f}$ Reaching the first mucosal incision of the duodenal bulb. $\mathbf{g}$ Submucosal dissection of lateral side when opening the pocket. $\mathbf{h}$ The mucosal defect in the stomach. $\mathbf{i}$ The mucosal defect in the duodenum. $\mathbf{j}$ The mucosal side of the resected specimen. The resected specimen is $36 \times 23 \mathrm{~mm}$ in size. $\mathbf{k}$ The submucosal side of the resected specimen. The central depression indicates the pyloric sphincter (arrows). I The yellow line indicates the border between the stomach and duodenum and red lines indicate the presence of carcinoma. $\mathbf{m}$ The pathology is a well- to moderately-differentiated intramucosal adenocarcinoma with negative horizontal and vertical margins and without lymphovascular invasion or ulceration. $\mathbf{n}$ The duodenal mucosa has a Brunner's gland (arrows). 

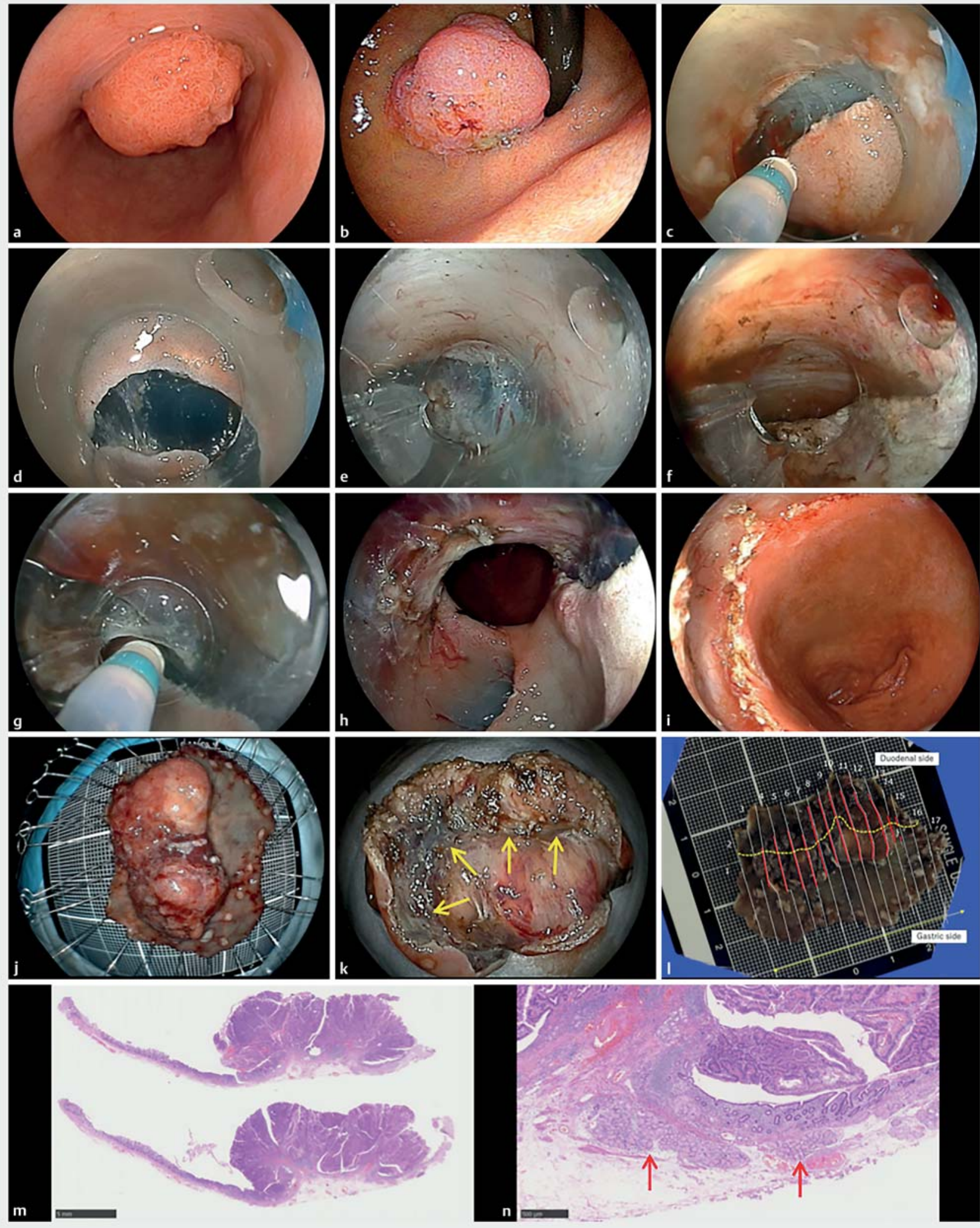

- Fig. 4 


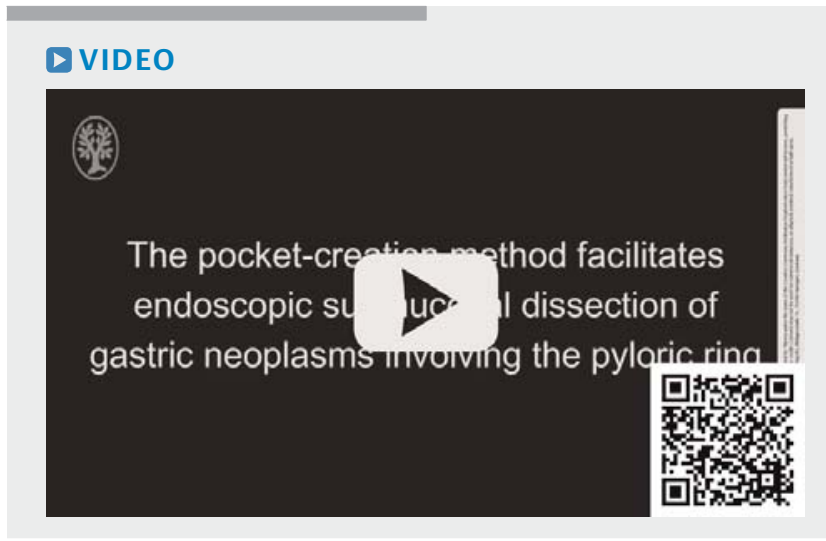

- Video 1 Procedure for endoscopic submucosal dissection of gastric neoplasms involving the pyloric ring with the pocket-creation method.
Gastric ESD from October 2006 to August 2019 $\mathrm{n}=2258$

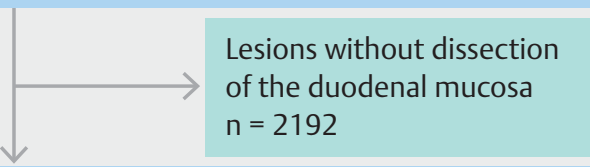

Lesions with dissection of the duodenal mucosa $n=66(2.9 \%)$

\begin{tabular}{c|c}
$\downarrow$ & $\downarrow$ \\
$\mathrm{PCM}$ & $\mathrm{CM}$ \\
$\mathrm{n}=20$ & $\mathrm{n}=46$
\end{tabular}

Fig. 5 Inclusion process for the study.

- Table 1 Baseline characteristics of patients.

\begin{tabular}{|c|c|c|c|}
\hline Characteristics & Pocket-creation method & Conventional method & $P$ value \\
\hline Number of lesions & 20 & 46 & \\
\hline Number of patients & 20 & 46 & \\
\hline Age, median (range), years & $73(70-79)$ & $76(68-79)$ & 0.818 \\
\hline Gender, male, n (\%) & $13(65)$ & $29(63)$ & 0.879 \\
\hline \multicolumn{4}{|l|}{ Tumor location, n (\%) } \\
\hline - Lesser curvature & $10(50)$ & $22(48)$ & 0.871 \\
\hline - Greater curvature & $2(10)$ & $4(9)$ & 0.865 \\
\hline - Anterior wall & $5(25)$ & $11(24)$ & 0.925 \\
\hline - Posterior wall & $3(15)$ & $9(19)$ & 0.659 \\
\hline \multicolumn{4}{|l|}{ Macroscopic type } \\
\hline - Elevated & $5(25)$ & $22(48)$ & 0.083 \\
\hline - Flat or depressed & $15(75)$ & $24(52)$ & \\
\hline \multicolumn{4}{|l|}{ Hood } \\
\hline - Cylindrical hood & $0(0)$ & $11(24)$ & 0.017 \\
\hline - Conventional ST hood & $15(75)$ & $21(46)$ & 0.028 \\
\hline - Short ST hood & $5(25)$ & $14(30)$ & 0.654 \\
\hline Antiplatelet or anticoagulation & $4(20)$ & $4(9)$ & 0.196 \\
\hline
\end{tabular}

statistical significance. No significant differences were observed in the incidence of adverse events. All delayed bleeding was successfully stopped by endoscopic hemostasis. No patient developed a stricture after ESD.

\section{Discussion}

This retrospective observational study demonstrates the usefulness of the PCM for ESD of gastric lesions involving the pyloric ring. In the PCM group, the $\mathrm{R} 0$ resection rate was significantly higher than that of the CM group. In the CM group, six specimens had positive vertical margins including three with a hole, while no specimen in the PCM group had these unfavorable events. We suspect that incorrect direction of the endoknife 
- Table 2 Clinical outcomes and adverse events.

\begin{tabular}{|c|c|c|c|}
\hline Characteristics & Pocket-creation method & Conventional method & $P$ value \\
\hline Tumor diameter, median (range), mm & $23(13-35)$ & $20(13-35)$ & 0.748 \\
\hline \multicolumn{4}{|l|}{ Pathologic findings, $\mathrm{n}(\%)$} \\
\hline - Adenoma & $0(0)$ & $1(2)$ & 0.506 \\
\hline - Intramucosal carcinoma & $17(85)$ & $41(89)$ & 0.637 \\
\hline - Slightly invasive $(<500 \mu \mathrm{m})$ submucosal carcinoma & $1(5)$ & $3(7)$ & 0.812 \\
\hline - Deeply invasive ( $\geq 500 \mu \mathrm{m})$ submucosal carcinoma & $2(10)$ & $1(2)$ & 0.161 \\
\hline - Ulcer & $2(10)$ & $0(0)$ & 0.029 \\
\hline - Vascular invasion & $2(10)$ & $1(2)$ & 0.161 \\
\hline - Lymphatic invasion & $1(5)$ & $1(2)$ & 0.538 \\
\hline - Venous invasion & $1(5)$ & $0(0)$ & 0.126 \\
\hline En bloc resection, $\mathrm{n}(\%)$ & $20(100)$ & $45(98)$ & 0.506 \\
\hline R0 resection, n (\%) & $20(100)$ & $38(83)$ & 0.047 \\
\hline Hole in resected specimen, $\mathrm{n}(\%)$ & $0(0)$ & $3(7)$ & 0.242 \\
\hline Positive horizontal margin, $\mathrm{n}(\%)$ & $0(0)$ & $3(7)$ & 0.242 \\
\hline Positive vertical margin, n (\%) & $0(0)$ & $6(13)$ & 0.090 \\
\hline Positive horizontal and vertical margin, n (\%) & $0(0)$ & $1(2)$ & 0.506 \\
\hline Resected specimen diameter, median (range), mm & $45(37-53)$ & $40(31-49)$ & 0.270 \\
\hline Dissection time, median (range), min & $75(58-111)$ & $75(50-115)$ & 0.856 \\
\hline Dissection speed, median (range), $\mathrm{mm}^{2} / \mathrm{min}$ & $16(12-25)$ & $11(8-20)$ & 0.148 \\
\hline Follow-up snare resection, $\mathrm{n}(\%)$ & $0(0)$ & $1(2)$ & 0.506 \\
\hline Delayed bleeding, n (\%) & $2(10)$ & $2(4)$ & 0.376 \\
\hline Perforation, n (\%) & $0(0)$ & $0(0)$ & 1.000 \\
\hline
\end{tabular}

caused by a firm pyloric sphincter damaged specimens being resected in the CM group ( $\vee$ Fig. 1 a). We believe that the PCM with the ST hood is a useful and suitable method to accomplish this difficult ESD by compressing the firm elevated sphincter muscle using both traction and counter-traction in the pocket.

In this study, ESD with the CM could not accomplish an R0 resection in eight patients $(17 \%)$. First, the mucosa being dissected gradually slides down into the duodenum making submucosal dissection quite difficult due to the downward view in the CM. In this case, snare resection during the last stage of ESD is necessary and adequate margins cannot be maintained. Using a snare may damage the specimen. Second, the CM cannot compress the pyloric sphincter because of the circumferential mucosal incision in the stomach. In getting over the pyloric ring, the endoscope approach becomes vertical to the sphincter and the tip of endoscope goes into the lesion resulting in cutting of the specimen being resected ( $\triangleright$ Fig. 1 ). Even if a hole is not in the specimen, a thick submucosal layer may not be obtained with the CM. Three resected specimens in the present study had positive vertical margins. However, the PCM accomplished an R0 resection of all lesions including two lesions with $\geq 500-\mu \mathrm{m}$ submucosal invasion by obtaining a thick submucosal layer.

According to the Japanese gastric cancer treatment guidelines, an $\mathrm{R} 0$ resection is essential for curative resection [8]. To estimate local curability and lymph node metastases, tumor size, tumor depth, lymphovascular invasion and vertical margin are important factors to determine next therapeutic strategy after ESD [9]. These are mainly based on factors relating to the submucosal layer, not the mucosal layer. Therefore, obtaining an adequate submucosal layer is important to obtain optimal clinical outcomes. The specimen resected by the PCM has a thick submucosal layer because the PCM enables endoscopists to select the dissection layer in the pocket by visualizing the submucosa and stabilizes the tip of the endoscope regardless of cardiac or respiratory movements. Applying both traction and counter-traction in the pocket provides clear visualization and stabilization. Further, unlike the CM, the PCM enables an antegrade approach without retroflexion by compressing the pyloric sphincter resulting in a straightforward approach without the need for special traction devices.

ESD of gastric lesions at the pyloric ring is challenging, even for expert endoscopists. Bae et al reported that a gastric tumor 
with duodenal extension is a significant factor leading to difficult ESD [10]. In the CM, the most difficult point is the downward facing view just after getting over the pyloric ring using an antegrade approach. A Korean study reported that the usefulness of the retroflexed approach in the duodenum for ESD of gastric lesions involving the pyloric ring improved the en bloc resection rate [11]. However, the retroflexed approach in the duodenal bulb suffers from limited maneuverability in a narrow lumen that may result in mucosal injury or perforation. Therefore, submucosal dissection using an antegrade approach compressing the sphincter muscle with the PCM is desirable.

We recognize the acknowledged limitations of this study. This is a single-center, retrospective observational study using historical controls. Evolution of devices and the learning curve may influence the outcomes. Long-term outcomes including local recurrence and survival were not evaluated. Although only expert endoscopists performed the ESDs in this study, technical experience may be a source of bias. This study did not include ESD with traction devices.

\section{Conclusions}

In conclusion, this is the first study reporting the advantages of the PCM for ESD of gastric lesions involving the pyloric ring. The PCM is a unique and suitable strategy for compressing the pyloric sphincter and enables an R0 resection. Use of the PCM with the ST hood is a viable option to accomplish ESD for superficial gastric neoplasms involving the pyloric ring.

\section{Competing interests}

Dr. Yamamoto is a consultant for Fujifilm Corporation and has received honoraria, grants, and royalties from the company. Drs. Miura, Sakamotor, and Hayashi have received honoraria and grants from FUJIFILM Medical Co., Ltd.

\section{References}

[1] Ferlay J, Colombet M, Soerjomataram I et al. Estimating the global cancer incidence and mortality in 2018: GLOBOCAN sources and methods. Int J Cancer 2019; 144: 1941-1953

[2] Konuma $\mathrm{H}$, Matsumoto $\mathrm{K}$, Ueyama $\mathrm{H}$ et al. Procedure time for gastric endoscopic submucosal dissection according to location, considering both mucosal circumferential incision and submucosal dissection. Gastroenterol Res Pract 2016: doi:10.1155/2016/9183793

[3] Abe S, Wu SYS, Ego M et al. Efficacy of current traction techniques for endoscopic submucosal dissection. Gut Liver 2020; 14: 673-684

[4] Miura Y, Shinozaki S, Hayashi Y et al. Duodenal endoscopic submucosal dissection is feasible using the pocket-creation method. Endoscopy 2017; 49: 8-14

[5] Hayashi Y, Sunada K, Takahashi $\mathrm{H}$ et al. Pocket-creation method of endoscopic submucosal dissection to achieve en bloc resection of giant colorectal subpedunculated neoplastic lesions. Endoscopy 2014; 46: (Suppl. 01): E421-422

[6] Hayashi Y, Shinozaki S, Sunada K et al. Efficacy and safety of endoscopic submucosal dissection for superficial colorectal tumors more than $50 \mathrm{~mm}$ in diameter. Gastrointest Endosc 2016; 83: 602-607

[7] Takezawa T, Hayashi Y, Shinozaki S et al. The pocket-creation method facilitates colonic endoscopic submucosal dissection (with video). Gastrointest Endosc 2019; 89: 1045-1053

[8] Japanese gastric cancer treatment guidelines 2018 (5th edition). Gastric Cancer 2020: doi:10.1007/s10120-020-01042-y

[9] Hatta W, Gotoda T, Oyama T et al. A Scoring system to stratify curability after endoscopic submucosal dissection for early gastric cancer: "eCura system". Am J Gastroenterol 2017; 112: 874-881

[10] Bae JH, Kim GH, Lee BE et al. Factors associated with the outcomes of endoscopic submucosal dissection in pyloric neoplasms. Gastrointest Endosc 2015; 81: 303-311

[11] Lim CH, Park JM, Park CH et al. Endoscopic submucosal dissection of gastric neoplasia involving the pyloric channel by retroflexion in the duodenum. Dig Dis Sci 2012; 57: 148-154 